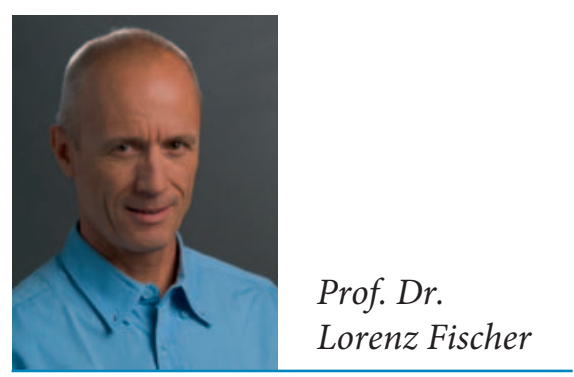

Die Sicht der Neuraltherapie

Herr Prof. Fischer, das zentrale Medikament der Neuraltherapie ist das Procain, das bereits seit 100 Jahren im Einsatz ist. Was gibt es Neues in Ihrem Fach?

Prof. Dr. Lorenz Fischer: Es hat grosse Fortschritte gegeben im Hinblick auf das Verständnis der Wirkung der Neuraltherapie auf neurophysiologischer Ebene. So können wir heute eine Kausalkette zwischen dem Wirkstoff und seiner Wirkung nachzeichnen. Mittlerweile erarbeiten verschiedene Forschergruppen auch ausserhalb der Neuraltherapie die weiteren Wirkungen der Lokalanästhetika. Die Ergebnisse dieser Forschung helfen uns, die Wirkmechanismen der Neuraltherapie besser zu verstehen. Wir wissen heute, dass das Procain unter anderem eine modulierende Wirkung auf das Immunsystem hat und zellstabilisierend ist, dass es antiarrhythmisch wirkt und die Zirkulation fördert. Am Grundprinzip der Neuraltherapie hat sich zwar nichts Wesentliches geändert, die Wirkmechanismen werden mithilfe der modernen Neurophysiologie jedoch besser verstanden.

\title{
Expertengespräch
}

Schweiz Z Ganzheitsmed 2015;27:322-327

DOI: 10.1159/000441908

\section{Jahre Komplementärmedizin an der Universität Bern}

Das Institut für Komplementärmedizin (IKOM), ursprünglich Kollegiale Instanz für Komplementärmedizin (KIKOM), der Universität Bern wurde 1995 aufgrund eines Regierungsratsbeschlusses errichtet. Es umfasst die Bereiche Anthroposophisch erweiterte Medizin, Klassische Homöopathie, Neuraltherapie sowie Traditionelle Chinesische Medizin. Die bisherige Dozentur für Anthroposophisch erweiterte Medizin mit einem Beschäftigungsgrad von $25 \%$ wurde 2014 in eine ausserordentliche Professur umgewandelt.

Bei der Gründung sah das Berner Konzept eine Unterteilung der neuen Instanz vor: Es wurde eine Stelle im Rang einer ausserordentlichen Professur geschaffen, diese jedoch zu je 25\% auf vier Dozenturen aufgeteilt war. Damit waren die vier häufigsten komplementärmedizinischen Fachgebiete der Schweiz gleichwertig an der Universität vertreten (in Klammern die Dozenten zum Zeitpunkt der Gründung): Klassische Homöopathie (Dr. André Thurneysen), Traditionelle Chinesische Medizin/Akupunktur (Dr. Brigitte Ausfeld-Hafter), Anthroposophische Medizin (Dr. Peter Heusser) und Neuraltherapie (Dr. Andreas Beck).

SeitderGründung derKIKOMhaben sich dieDozentenund dieBeschäftigten verjüngt; das Konzept ist jedoch bis heute erhalten geblieben. So wird die Klassische Homöopathie heute von Dr. med. Martin Frei-Erb, die Traditionelle Chinesische Medizin/Akupunktur von Dr. med. Johannes Fleckenstein, die Anthroposophisch erweiterte Medizin von Prof. Dr. med. Ursula Wolf und die Neuraltherapie von Prof. Dr. med. Lorenz Fischer geleitet, die nachfolgend in Einzelinterviews zu Wort kommen. Im September 2013 wurde die KIKOM dann zum IKOM der Universität Bern aufgewertet.

Das IKOM ist gemäss dem Reglement über die Organisation der Medizinischen Fakultät der Universität Bern verantwortlich für Lehre, Forschung, Dienstleistungen, Nachwuchsförderung und - soweit möglich - für Weiterund Fortbildungen. Folgende Quellen sind zur Finanzierung benannt: Mittel der Universität, sonstige Drittmittel und die Erträge durch Dienstleistungen. Die vier Fachbereiche organisieren sich gemeinsam über das IKOM, sind in der Ausübung und Durchführung ihrer Aufgaben jedoch unabhängig.

\section{Wie würden Sie einem Kollegen, der von der Neuraltherapie keine Ahnung hat, Ihr Gebiet beschreiben?}

Es handelt sich um eine Methode, die mit Injektionen (Lokalanästhetika) arbeitet und mit der man die Regulationsmöglichkeiten des Nervensystems nutzen kann. Die Neuraltherapie ist hervorragend geeignet, akute und chronische Schmerzen so- wie funktionelle Störungen zu behandeln. Das Nervensystem kann sich pathophysiologisch so verändern, dass Schmerzimpulse einfacher weitergeleitet und gespeichertwerden (Schmerzgedächtnis, sogenannte Neuroplastizität). Solche Veränderungen sind prinzipiell reversibel und können durch Anwendung von Lokalanästhetika «gelöscht» werden - mit nachfolgender Neuorganisation der neuronalen Strukturen. 


\section{Ist die Neuraltherapie \\ eine Methode der \\ Komplementärmedizin, die \\ man kausal gut versteht?}

Was die Kausalität betrifft, haben Sie recht; wir kennen die Wirkung zum Teil schon genau. Die Neuraltherapie als reine Methode der Komplementärmedizin zu beschreiben, trifft die Sache aber nicht mehr richtig, da sie mittlerweile in der Schulmedizin angekommen ist. Sie ist vollkommen akzeptiert und wird ganz selbstverständlich in Schmerzpraxen und -kliniken im lokalen und segmentalen Bereich als sogenannte «diagnostische und therapeutische Lokalanästhesie» eingesetzt. Studien belegen die Wirksamkeit, Zweckmässigkeit und Wirtschaftlichkeit (WZW) der Neuraltherapie. Ein Blick in die Leistungsverordnung der obligatorischen Krankenpflegeversicherung (OKP) zeigt, dass die Neuraltherapie (lokal und segmental) definitiv in die KrankenpflegeLeistungsverordnung (KLV) aufgenommen wurde (ohne Notwendigkeit einer weiteren Evaluation).

\section{Ist diese Akzeptanz das \\ Ergebnis einer längeren \\ Entwicklung oder hat es einen plötzlichen Durchbruch gegeben?}

Sie ist das Ergebnis einer längeren Entwicklung. Im Laufe der Zeit hat die Schulmedizin vieles von der Neuraltherapie übernommen. Vor allem in der Schmerztherapie hat sie Einzug gehalten, und die Indikationen sind stetig erweitert worden. Daneben wurden in den vergangenen Jahren immer mehr überzeugende Studien publiziert.

\section{Wie muss man sich die Wirkung der Neuraltherapie vorstellen?}

In den letzten Jahren wurde viel über die Neuroplastizität, also z.B. über das Gedächtnis des Nervensystems, geforscht. Dabei ist klar geworden, dass man dieses Gedächtnis oft auch «löschen» kann. Durch den gezielten Einsatz von Procain und ähnlichen Mitteln an Nerven, Nervenumschaltstellen, in Triggerpunkten, Gelenken usw. ist das prinzipiell möglich. Wir stellen einige Strukturen im Nervensystem wieder auf «Null», drücken also sozusagen den «Reset»-Knopf und geben dem System damit die Möglichkeit, die Reaktion auf bestimmte Reize wieder neu zu lernen, sich wieder neu zu organisieren. Daneben «lernen» bestimmte Nerven nach Umflutung mit Lokalanästheti$\mathrm{ka}$, weniger Entzündungssubstanzen auszuschütten.

\section{Welche Rolle hat das IKOM (früher KIKOM) in den letzten Jahrzehnten gespielt, um das Fach zu etablieren?}

Das IKOM besteht aus vier Fachrichtungen. Die Dozenten aller Fachrichtungen waren am «Programm Evaluation Komplementärmedizin» des Bundesamtes für Gesundheit beteiligt. Im Falle der Neuraltherapie waren es einerseits kontrollierte Studien unter der Leitung von Professor André Busato und mir und andererseits ein 300-seitiger «Health Technology Assessment»-Bericht (Leitung: Lorenz Fischer). Soeben wurde von der Dozentur Neuraltherapie am IKOM eine grosse Studie mit sehr guten Langzeitergebnissen bei überwiesenen therapieresistenten Schmerzpatienten publiziert (Egli $S$, et al: Long-term results of therapeutic local anesthesia (neural therapy) in 280 referred refractory chronic pain patients. BMC Complement Altern Med 2015;15:200).

\section{Wenn Sie die Zeit seit Ihrem Studium bis heute Revue passieren lassen, wie haben sich Akupunktur und Chinesische Medizin entwickelt?}

Als ich begann, mich mit der Akupunktur zu beschäftigen, war sie innerhalb der Schulmedizin bereits salonfähig geworden, und die Kämpfe um universitäre Anerkennung, die noch einige Jahre früher ausgetragen wurden, habe ich nicht mehr miterlebt. Seitdem ist eine solide Basis an wissenschaftlicher Evidenz entstanden. Dazu gehören zum einen das Verständnis über Grundlagen 
und Wirkmechanismen, zum anderen Studien über die Wirksamkeit der Verfahren. Gerade das wissenschaftliche Verständnis der Methode hat grosse Fortschritte gemacht. Die Zahl der Publikationen ist geradezu explodiert, und jedes Jahr erscheinen etwa 500 Artikel, die sich in irgendeiner Form mit Akupunktur und Chinesischer Medizin beschäftigen und auf PubMed (einer Bibliothek international anerkannter Fachzeitschriften) gelistet sind. Darüber hinaus gibt es noch unzählige Veröffentlichungen, die in sonstigen Zeitschriften erscheinen.

\section{Kann man von einem Wandel sprechen, was die Haltung der Schulmedizin gegenüber der Akupunktur und Chinesischen Medizin betrifft?}

Auf alle Fälle, denn nicht nur in Praxen oder kleinen Spitälern, sondern auch in Universitätskliniken ist es heute selbstverständlich, dass die Akupunktur Eingang in die Behandlungsschemata findet. Man hat gesehen, dass sich Akupunktur und Chinesische Medizin sehr gut mit der Schulmedizin kombinieren lassen. In der Therapievon chronischen Schmerzen wird beispielsweise ein multimodales Vorgehen empfohlen, in welches sich die Akupunktur hervorragend integrieren lässt. Auch in anderen Fächern der Medizin merkt man, dass es nicht darum geht, eine «einzig» wirksame Therapie zu finden. Stattdessen hat sich ein Spektrum verschiedener Therapieansätze bewährt, das dem $\mathrm{Pa}$ tienten in seinem Gesamterleben der Krankheit hilft, die Symptome lindert und letztlich $\mathrm{zu}$ einer Überwindung der Krankheit beiträgt. Was wir zurzeit sehen, ist ein Paradigmenwechsel von der Monotherapie hin zu multimodalen Therapien. Dabei ist die Akupunktur ein sinnvoller Bestandteil.

Diese veränderte Einstellung ist ein Ergebnis der letzten 20 Jahre. Mittlerweile haben wir junge moderne Chefärzte und Lehrstuhlinhaber an den

Universitäten, die der Akupunktur und der Chinesischen Medizin gegenüber sehr offen sind. Man darf dabei jedoch nicht die Vorreiterrolle einzelner Universitäten vergessen: In München gibt es seit über 35 Jahren ein entsprechendes Wahlfach Akupunktur für Studenten, gerade weil der damalige Ordinarius dafür offen war und dieses Angebot ermöglicht hat. In Bern wurde vor 20 Jahren die KIKOM (jetzt IKOM) gegründet, um diese Methoden $\mathrm{zu}$ lehren und $\mathrm{zu}$ erforschen. Heutzutage gibt es viele weitere Universitäten, an denen entsprechende Veranstaltungen stattfinden, und an den meisten Universitäten findet man mindestens eine Forschungsgruppe, die schon mal eine Arbeit zum Thema Akupunktur durchgeführt hat.

\section{Wie kann man bei der Akupunktur die Wirkung der «echten» Behandlung von der Placebo-Wirkung unterscheiden?}

Das Wort «Placebo» würde ich nicht gebrauchen, sondern von Scheinbehandlungen sprechen. Bei denen lässt sich auch eine Wirkung feststellen, was einen recht schnell zum Thema spezifische Wirkeffekte bringt. Das ist die Frage, was eine Therapie eigentlich ausmacht. Wir wissen mittlerweile in der Medizin, dass ganz starke Behandlungseffekte allein schon durch den therapeutischen Rahmen auftreten, also durch die Beziehung, die Arzt und Patient eingehen. Das ist ein wesentlicher Grund, weshalb manche Therapien bei manchen Ärzten besser funktionieren als bei anderen. Ein Teil der Wirksamkeit ist durchaus unabhängig davon, wo der Akupunkteur sticht, sondern abhängig von der guten Beziehung, die zwischen Arzt und Patient aufgebaut wird.

Aus einer grossen Meta-Analyse mit über 18000 Patienten weiss man jedoch, dass es bei der Akupunktur tatsächlich einen Unterschied zwi- schen einer Scheinbehandlung und dem Setzen der Nadel am richtigen Ort gibt. Die richtige Akupunktur wirkt besser und länger anhaltender. Grundlagenarbeiten zeigen zudem, dass bestimmte physiologische Reaktionen umso stärker ausgelöst werden, je präziser man einen Akupunkturpunkt «behandelt».

\section{Ist die Beziehungsqualität ein sehr ernstzunehmender und wesentlicher Einflussfaktor für den Erfolg einer Therapie wie der Akupunktur?}

Sie ist ein sehr ernstzunehmender Einflussfaktor, der aber auch bei anderen Therapien eine Rolle spielt. Das Anfassen und das Berühren sollte ein wesentlicher Teil in der medizinischen Diagnostik und Therapie sein und hat einen grossen Einfluss auf den Behandlungserfolg. Es gibt eine schöne Studie von der Harvard Medical School, die gezeigt hat, dass bereits die Empathie bei sonst gleicher Behandlung dazu beiträgt, dass Patienten die Schwere eines Symptoms geringer empfinden.

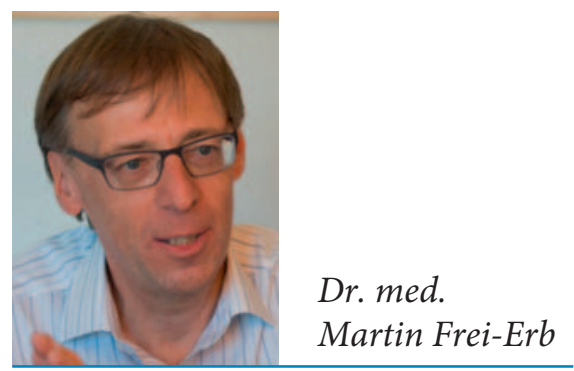

Die Sicht der Homöopathie

\section{Herr Dr. Frei-Erb: Was waren in den letzten 20 Jahren die grössten Fortschritte, welche die Homöopathie gesehen hat?}

Dr. med. Martin Frei-Erb: Die Gründung der KIKOM war sehr wichtig, und ein wirklicher Meilenstein war die Publikation einer Studie im Jahr 
2005 über die homöopathische Behandlung von Kindern und Jugendlichen mit Aufmerksamkeitsdefizitsyndrom und Hyperaktivität (ADHS). Dort wurde gezeigt, dass die Kinder und Jugendlichen tatsächlich von einer solchen Behandlung profitieren. Seitdem ist diese Indikation ein Forschungsschwerpunkt in der Fachrichtung Homöopathie. Sehr wichtig ist, dass wir mittlerweile einen Langzeitlauf haben und einige Patienten seit über 10 Jahren verfolgen können. Diese Studie zeigt, dass wir bei der Behandlung des ADHS mit homöopathischen Verfahren auf dem richtigen Weg sind. Wir bleiben bei dieser Forschung am Ball und planen eine Nachfolgestudie mit Verfahren der Bildgebung. Wir wollen wissen, was im Gehirn der Patienten unter den verschiedenen Behandlungsregimen passiert. Es geht vor allem um Kinder und Jugendliche, die homöopathisch behandelt werden.

\section{War die Forschung in der Homöopathie auch international erfolgreich?}

Die Beteiligung am Forschungsprogramm CAMbrella hat der Homöopathie viel gebracht. Wir beteiligten uns während 3 Jahren daran, von 2010 bis 2012. Dabei handelte es sich um ein paneuropäisches Forschungsprojekt, in dem 16 Universitäten aus 12 europäischen Ländern ein Netzwerk geknüpft haben. Dort beschäftigten wir uns in 8 Arbeitsgruppen mit ganz unterschiedlichen Fragen, wie z.B. einer Definition der Komplementärmedizin. Man hat nach langen und schwierigen Diskussionen einen Konsens gefunden, der ausführlicher als die amerikanische Definition ist.

In einigen europäischen Ländern sind bestimmte Methoden der Komplementärmedizin in die nationalen Gesundheitssysteme integriert worden. Eine Arbeitsgruppe hat sich um die juristischen Aspekte gekümmert. Eine andere hat sich damit beschäftigt,

was die Patienten in Europa von der Komplementärmedizin erwarten. Das Resultat ist eine Roadmap für die Forschung zur Komplementärmedizin in Europa.

\section{Ist es ungewöhnlich, dass die Europäische Union (EU) und andere Forschungsförderer überhaupt Forschung in der Komplementärmedizin finanzieren, oder ist das ein Zeichen eines Zeitenwandels?}

Es ist auf alle Fälle ein Zeichen, dass sich die alten Fronten auflösen. Im Moment muss man abwarten, wie sich die Förderung in Zukunft entwickelt. Von der EU sind Signale gekommen, dass es im Forschungsprogramm «Horizon 2020» kein rein komplementärmedizinisches Forschungsprojekt mehr geben wird. Wir müssen uns mit der konventionellen Medizin bei gewissen Themen zusammenschliessen. Und da gibt es natürlich interessante Themen, wie etwa die Schmerzbehandlung bei chronischen Schmerzen oder die Antibiotikaresistenzen. Allein schon ein Projekt auszuarbeiten und einzureichen, ist aber eine grosse Herausforderung und braucht viel Anstrengungen, um dann auch erfolgreich zu sein.

\section{Hat sich die Reaktion der klassischen schulmedizinischen Kollegen gegenüber der Homöopathie geändert?}

Ich bin Hausarzt in Thun und dort im Kollegenkreis immer gut integriert gewesen. Mir wurde mit grosser Offenheit begegnet. In den Spitälern erlebe ich durch den Generationenwechsel ein gewisses Interesse an Komplementärmedizin und Homöopathie. Das wird auch dadurch beflügelt, dass Klinikdirektoren einfach gemerkt haben, dass die Patienten diese Methode wünschen. Es geht nicht mehr darum, den Patienten das eigene
Weltbild überzustülpen, sondern auch darum, das anzubieten, was sie wünschen. Bei mir in meiner Praxis ist das Interesse der Patienten seit über 22 Jahren unverändert gross.

\section{Ist bei den Patienten \\ die Bereitschaft, komplementärmedizinische Methoden «auszuprobieren», relativ weit verbreitet?}

Ja, und das wissen wir sehr genau aus Gesundheitsbefragungen, die wir an der KIKOM ebenfalls gemacht haben. Eine der Umfragen ist eine Dissertation auf der Kinderonkologie, in der 10 Jahre retrospektiv alle Patienten der Kinderonkologie in Bern zur Nutzung der Komplementärmedizin befragt wurden. Dabei ging es nicht nur um die Homöopathie, sondern um die Frage, welche Methoden der Komplementärmedizin sie angewendet haben. Uns hat interessiert, wieso sie verwendet wurden. Hier in Bern wendet die Hälfte der betroffenen Patienten und Familien Methoden der Komplementärmedizin an. Am häufigsten wird die Homöopathie verwendet, dann folgt die Einnahme von Vitaminen und Mineralstoffen. Der Hauptgrund ist die Linderung der Nebenwirkungen. Es geht aber auch um eine allgemeine Unterstützung im Behandlungs- und Heilungsprozess.

Die Umfrage zeigt, dass die Schulmediziner keine Angst haben müssen, dass ihre Patienten in die Alternativmedizin abrutschen und bei einer Krebserkrankung keine konventionelle Therapie machen. Wahrscheinlich ist die Compliance sogar noch besser, wenn neben dem Kinderonkologen oder dem Erwachsenenonkologen auch ein Komplementärmediziner beigezogen wird. Dazu braucht es aber weitere begleitende Untersuchungen. 


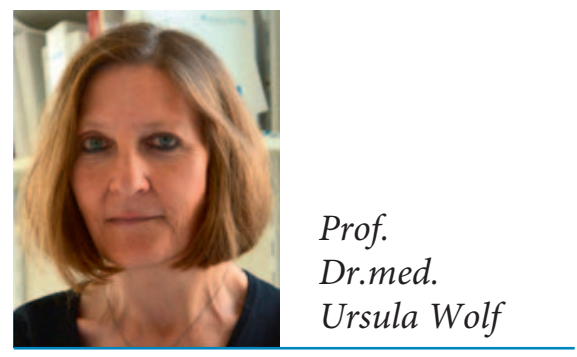

\section{Die Sicht der Anthroposophisch erweiterten Medizin}

\section{Frau Professor Wolf, Ihre Professur in Bern ist im Oktober 2014 neu installiert worden. Was sind Ihre Ziele?}

Prof. Dr. med. Ursula Wolf: Die Ziele der Professur sind, in der Komplementärmedizin und insbesondere der Anthroposophisch erweiterten Medizin die Forschung und Lehre zu stärken sowie diese Disziplinen stärker akademisch zu verankern.

\section{Die Professur ist mit Unterstützung von Partnern zustande gekommen. Tangiert das die wissenschaftliche Unabhängigkeit?}

Das ist eine verständliche Frage. Die Universität Bern hat klare im Universitätsgesetz verankerte Richtlinien für die Unterstützung von Lehrstühlen durch externe Partner. So wird vertraglich festgehalten, dass die Freiheit von Forschung und Lehre gewährleistet ist. Auch für mich persönlich ist das eine unerlässliche Voraussetzung für diese Professur. Das Modell der Unterstützung durch externe Partner ist übrigens nicht neu. In der Schweiz gibt es mehrere solcher Professuren - und auch in den USA, wo es eine besondere Ehre ist, auf eine solche Professur gewählt zu werden. Das Besondere dieser Professur ist, dass es vier und nicht nur ein Partner sind, namentlich die Universität Bern, der Verein zur Förderung anthroposophischer Institutio- nen, die Firma Weleda und die Software AG-Stiftung.

\section{Wie profitieren die Forschung und die Partner von diesem Modell?}

Die Partner profitieren ideell, denn sie unterstützen die Professur. Dadurch, dass nun die Forschungsaktivitäten intensiviert werden, ist auch eine Leistungssteigerung zu erwarten. Das heisst, es werden in grösserem Masse als bisher Forschungsresultate erzielt werden, die relevant und nutzbringend sowohl für die Forschergemeinschaft als auch für die Gesellschaft und letztlich für die Patienten sein können. Ausserdem wird die Nachwuchsförderung gesteigert, indem eine grössere Anzahl von wissenschaftlichen Mitarbeitenden an der Professur arbeiten wird und mehr Studierende mit Masterarbeiten und Dissertationen als bisher betreut werden können.

\section{In welcher Richtung wollen Sie in Zukunft forschen?}

Das übergeordnete Ziel meiner Forschung ist es, auf hohem Niveau und führend zur Erweiterung des Wissens im Bereich der komplementären und Anthroposophisch erweiterten Medizin und letztlich zur Verbesserung der medizinischen Versorgung für unsere Patienten beizutragen. Meine Forschungsschwerpunkte sind die Untersuchung der Wirksamkeit, Sicherheit und Wirkungsarten von Arzneimitteln und nichtmedikamentöser Therapien sowie die Entwicklung von multimodaler Forschungsmethodologie.

So untersuchen wir aktuell beispielsweise in Kooperation mit der Universitätskinderklinik Bern mit einer doppelt verblindeten randomisierten Studie die Anwendung eines anthroposophisch-medizinischen Arzneimittels in der Neonatologie oder in Kooperation mit pneumologischen Zentren im In- und Ausland die
Effekte von anthroposophisch-medizinischer Sprachtherapie bei Patienten mit Asthma bronchiale auf quantitativ gemessene Lungenfunktionsparameter und die krankheitsbezogene Lebensqualität.

\section{Gehört das Vorurteil, dass sich Verfahren der Anthroposophischen Medizin nicht mit naturwissenschaftlichen Methoden untersuchen lassen, der Vergangenheit an?}

Ja, und es ist, wie Sie sagen, ein Vorurteil. Gerade in der Anthroposophisch erweiterten Medizin, deren Grundkonzept die Integration von natur- und geisteswissenschaftlichen Aspekten ist, konnte man immer schon mit naturwissenschaftlichen Methoden forschen. Also: Grundsätzlich lassen sich Verfahren der Anthroposophischen Medizin auch nach WZWKriterien untersuchen. Wichtig ist es, das Studiendesign der Forschungsfrage anzupassen, d.h., man muss sich fragen, ob man mit dem gewählten Ansatz wirklich das untersuchen kann, was man untersuchen möchte. In der Anthroposophisch erweiterten Medizin können alle Studiendesigns einsetzt werden.

\section{Wie gross ist das Interesse von Studierenden an Ihrem Fach?}

Die Universität Bern ist im Hinblick auf die Integration von komplementärmedizinischen Themen in die Ausbildung von Ärztinnen und Ärzten führend in der Schweiz. Einige unserer Lehrveranstaltungen sind Teil des Pflicht-Curriculums. Das Interesse der Studierenden ist prinzipiell gross. Das inhaltlich sehr gedrängte Curriculum bedeutet für die Studierenden, sich hauptsächlich auf die Fächer der Standardmedizin konzentrieren zu müssen. Unsere Vorlesungen und anderen Lehrveranstaltungen 
sind in der Regel gut besucht. Die Masterarbeits- und Dissertationsthemen, die ich ausschreibe, sind meistens rasch vergeben.

\section{An welchen Themen arbeiten Masterstudierende oder} Doktoranden bei Ihnen derzeit?

Masterarbeitsstudierende und Dissertanten werden unter Berücksichtigung ihrer Präferenzen und Fähigkei- ten an allen Phasen eines Forschungsprojekts beteiligt. Damit erfahren die Studierenden nicht nur etwas zu dem Thema, an dem sie arbeiten, sondern lernen auch, wie man forscht. Diese Fähigkeit ist ihnen auch in ihrer weiteren Karriere nützlich. Masterarbeitsstudierende und Dissertanten sind beispielsweise bei den Forschungsprojekten zur Grundlagenforschung von anthroposophisch-medizinischer Sprachtherapie und der Anwendung dieser Therapie bei Patienten mit Asthma bronchiale, zu farbigem Licht auf den menschlichen Organismus, zu Effekten unter Misteltherapie und zu Effekten anthroposophischmedizinischer Arzneimittel mittels UV-Spektroskopie beteiligt. Dabei arbeiten die Studierenden an Literaturübersichtsartikeln oder bei experimentellen Arbeiten mit.

Interviews: Oliver Klaffke 